\title{
Special issue on matheuristics
}

\author{
Yannick Kergosien ${ }^{1}$ - Jorge E. Mendoza ${ }^{2}$. Vincent T'kindt ${ }^{1}$
}

Received: 22 February 2021 / Revised: 23 February 2021 / Accepted: 23 February 2021 /

Published online: 11 March 2021

(c) The Author(s), under exclusive licence to Springer Science+Business Media, LLC, part of Springer Nature 2021

Matheuristics are optimization techniques which make use of mathematical programming to improve the search of a good solution. Thanks to the recent improvement in the performance of mathematical optimization tools (e.g., black box solvers), matheuristics have become a go-to alternative to solve hard problems both in academia and industry. This special issue groups 10 contributions showing the versatility, efficiency, and effectiveness of this new breed of algorithms to solve a wide range of difficult problems.

Rossi, Singh and Sevaux introduce a column generation-based matheuristics to maximize the lifetime of a wireless sensor network made up of video cameras. In a nutshell, the problem consists in optimally selecting the activation time, the target direction, and the focal distance of each camera. After presenting some basic context in optics and video camera surveillance that are not necessarily familiar to the optimization community, the authors analyse the problem and derive theoretical properties that lay the foundation of their solution approach. Next, the authors formulate the problem as a linear program that they solve using column generation. Their model is based on the notion of groups. A group is a subset of active sensors, and for each active sensor, a direction and a focal distance. In the master problem, the authors select the number of time units that each group will be active making sure that the battery life constraints are met. Since the number of potential groups rapidly increases with problem size, the pricing suproblem generates interesting groups on the fly. Because the pricing subproblem is a combinatorial optimization problem, the authors solve it using a genetic algorithm. The latter is build using both standard and problem-specific operators. The authors report experiments carried on a rich set of 180 instances. They compare the results delivered by the approach to two upper bounds. Their analysis suggests that

\footnotetext{
$凶 \quad$ Jorge E. Mendoza

jorge.mendoza@hec.ca

Yannick Kergosien

yannick.kergosien@univ-tours.fr

Vincent T'kindt

vincent.tkindt@univ-tours.fr

1 Université de Tours - LIFAT, Tours, France

2 HEC Montréal, Montreal, Canada
} 
their matheuristic is able to deliver optimal solutions in most of the instances and produce results with small optimality gaps in the remaining ones.

Grangier, Gendreau, Lehuédé and Rousseau combine a large neighborhood search (LNS) and branch-and-check (BC) to solve a vehicle routing problem with crossdocking and resource constraints (VRPCD-RC). In their problem, they consider a set of transportation requests, each having different origins and destinations. Each vehicle starts from the depot, performs a pickup leg which ends at the cross-dock, then it performs a delivery leg and returns to the depot. At the cross-dock, some transportation requests are exchanged between vehicles, creating precedence constraints between pickup and deliveries legs of different vehicles. They consider that the number of doors that can be processed simultaneously at the cross-dock is limited. To solve the problem they introduce a matheuristic following the route-first, assemble-second principle. In the routing phase they use a LNS to generate a pool of routes and a pool of legs. In the assembling phase a BC procedure is use to recombine routes and legs from the pools to find high-quality solutions. The authors report experiments on instances ranging from 50 to 200 requests from the literature. They analyze the behavior of their method running under different configurations and practical settings.

Dupin and Talbi introduce a matheuristic to deal with a simplified version of the refueling and maintenance planning problem introduced by French electricity giant EDF for the 2010 ROADEF/EURO Challenge. To solve this challenging and relevant industrial problem, the authors propose several constructive matheuristics and a variable neighborhood descent local search using mixed integer programming. In addition to showing the efficiency of the proposed solution methods, the computational experiments allowed the authors to explain some results of the Challenge and to analyze some previous proposed approaches which use mathematical models and local search heuristics.

Lahrichi, Deroussi, Grangeon and Norre address a scheduling problem in the the context of the 4.0 industry. The problem is called reconfigurable transfer line balancing problem. It consists in assigning operations to workstations and sequencing them on each workstation subject to several constraints: sequence-dependent setup times, precedence, inclusion, exclusion and accessibility constraints. The authors propose an efficient matheuristic following the principle of balance-first, sequence-last (BFSL). The proposed method has two phases, a constructive phase and an improvement phase. The method is based on linear programming, dynamic programming and a simulated annealing. The computational experiments show that their matheuristic outperforms a genetic algorithm from literature.

Yang and Leus focus on the solution of a two-stage hybrid flowshop scheduling problem with time windows. The goal is to schedule the jobs to minimize the weighted sum of their completion times. This scheduling problem, which relates to the optimization of production systems, is a hard and complex problem but efficiently solved by means of a tricky local branching heuristic. Computational experiments highlight the efficiency of that matheuristic.

Della Croce, Grosso, and Salassa consider the solution of the two-machine noidle and no-wait flowshop scheduling problem with minimization of the sum of job completion times. They propose several structural properties of the problem and position-based IP formulation of the problem. The proposed heuristic algorithm first 
generate initial solutions improved by a local search procedure embedding the structural properties. On the best computed solution is finally applied a very large scale neighborhood search based on the IP formulation. Computational experiments show the efficiency of the global procedure and the contribution of the very large scale neighborhood search.

Another scheduling problem is dealt with by Ahmadian and Salehipour who consider the scheduling of a just-in-time production system in a jobshop setting. The objective is to schedule jobs, each having its own routing, so that the weighted deviation to due dates is minimized. The proposed matheuristic relies on a decomposition of the problem into smaller sub-problems solved by means of an IP formulation. Computational experiments show that the proposed matheuristic provides better solutions than those obtained by solving an IP formulation of the global problem but with a limited CPU time.

Chandrasekharan, Smet, and Wauters tackle the problem of scheduling tasks to multi-skilled employees with the goal of minimizing the number of required employees. An assignment based IP formulation is proposed and integrated into a constructive matheuristic. This one proceeds by decomposing the problem into sub-problems solved iteratively by means of the IP formulation. Computational experiments report the efficiency of the matheuristic depending on the used problem decomposition.

Ferrer, Chicano, and Toro consider the problem of selecting products and features to test in the context of software production lines. The aim of this optimization problem is not only to select the products to test but also to determine the order in which they are tested. A matheuristic called Construct, Merge, Solve and Adapt is proposed and compared to four other heuristics from the literature, including other matheuristics. Computational experiments show that the proposed matheuristic outperforms the stateof-the-art heuristics.

In a theoretically oriented work, Guignard and Ahlatcioglu present a matheuristic called the Convex Hull Heuristic $(\mathrm{CHH})$ to find good feasible solutions to mixed integer programming problems with a nonlinear objective function and linear constraints. The $\mathrm{CHH}$ is based on the mathematical programming algorithm called simplicial decomposition. The main idea of the proposed method is to alternate between the resolution of nonlinear continuous problem and a linearized $0-1$ problem to generate a new extreme point of the integer convex hull of the feasible set. The authors test the $\mathrm{CHH}$ on 4 types of quadratic $0-1$ optimization problems: the generalized quadratic assignment problems, the cross-dock door assignment problems, the quadratic assignment problems and the quadratic knapsack problems (QKP).

This special issue is the result of the work of many people. We would like to acknowledge the work of all the referees who contributed their time to review the 40 submissions we received. We also want to sincerely thank Pr. Manuel Laguna for giving us the opportunity to prepare this issue, and for his valuable assistance and guidance during the process.

Publisher's Note Springer Nature remains neutral with regard to jurisdictional claims in published maps and institutional affiliations. 